\title{
Coronal O vI emission observed with UVCS/SOHO during solar flares: Comparison with soft X-ray observations
}

\author{
S. Mancuso ${ }^{1}$, S. Giordano ${ }^{1}$, and J. C. Raymond ${ }^{2}$ \\ ${ }^{1}$ Istituto Nazionale di Astrofisica, Osservatorio Astrofisico di Torino, Strada Osservatorio 20, 10025 Pino Torinese, Italy \\ e-mail: mancuso@oato.inaf.it \\ 2 Harvard-Smithsonian Center for Astrophysics, 60 Garden Street, MS 50, Cambridge, MA 02138, USA
}

Received 22 July 2015 / Accepted 12 April 2016

\begin{abstract}
In this work, we derive the O VI $1032 \AA$ luminosity profiles of 58 flares, during their impulsive phase, based on off-limb measurements by the Ultraviolet Coronagraph Spectrometer (UVCS) aboard the SOlar and Heliospheric Observatory (SOHO). The O VI luminosities from the transition region plasma (here defined as the region with temperatures $5.0 \leq \log T(\mathrm{~K}) \leq 6.0$ ) were inferred from the analysis of the resonantly scattered radiation of the $\mathrm{O}$ VI coronal ions. The temperature of maximum ionization for O VI is $\log T_{\max }(\mathrm{K})=5.47$. By comparison with simultaneous soft X-ray measurements, we investigate the likely source (chromospheric evaporation, footpoint emission, or heated prominence ejecta) for the transition region emission observed during the impulsive phase. In our study, we find evidence of the main characteristics predicted by the evaporation scenario. Specifically, most O VI flares precede the X-ray peaks typically by several minutes with a mean of $3.2 \pm 0.1 \mathrm{~min}$, and clear correlations are found between the soft X-ray and transition region luminosities following power laws with indices $\sim 0.7 \pm 0.3$. Overall, the results are consistent with transition region emission originating from chromospheric evaporation; the thermal X-ray emission peaks after the emission from the evaporation flow as the loops fill with hot plasma. Finally, we were able to infer flow speeds in the range $\sim 20-100 \mathrm{~km} \mathrm{~s}^{-1}$ for one-third of the events, 14 of which showed speeds between 60 and $80 \mathrm{~km} \mathrm{~s}^{-1}$. These values are compatible with those found through direct spectroscopic observations at transition region temperatures by the EUV Imaging Spectrometer (EIS) on board Hinode.
\end{abstract}

Key words. Sun: activity - Sun: corona - Sun: coronal mass ejections (CMEs) - Sun: flares - Sun: UV radiation

\section{Introduction}

The formation of large solar flares typically involves an impulsive phase followed by a gradual phase. The impulsive phase is attributed to the rapid downward acceleration of particles at the reconnection region, where magnetic energy is mainly transformed into kinetic energy of non-thermal particles, which then deposit their energy in the chromosphere and produce hard X-ray emission at the loop footpoints. The downward flux of accelerated particles generates a localized pressure pulse that drives an evaporation upflow of heated plasma into the corona, which then gradually cools as it radiates in soft X-ray and extreme ultraviolet (EUV) wavelengths. The gradual phase is thus associated with the longer lasting soft X-ray emission from chromospheric plasma evaporated into the corona. This two-phase process is often confirmed by observations that show the bulk X-ray and EUV coronal emissions delayed with respect to the impulsively rising hard X-ray, UV, and optical emissions from the lower atmosphere.

Ultraviolet spectra during the impulsive phase of solar flares are rarely available. This is due to the limited field of view of the spectrographs making raster scans and because the spectrometer slit must be serendipitously positioned at the right time in the right place although the impulsive phase usually occurs in a small region and only lasts a short time. Therefore, most available spectroscopic observations of flares pertain to the gradual phase. During the impulsive phase of solar flares, the nature of the emission from UV spectral lines generated in the transition region such as the O VI $1032 \AA$ line, which is formed at about $3 \times 10^{5} \mathrm{~K}$ near the peak of the radiative cooling curve, is thus poorly known. In some favorable cases, however, the flare's signature can be detected indirectly in observations of resonantly scattered radiation from $\mathrm{O}$ VI ions higher up in the corona. During its activity, the Ultraviolet Coronagraph Spectrometer (UVCS; Kohl et al. 1995) aboard the SOlar and Heliospheric Observatory (SOHO) recorded several observations of O VI $1032 \AA$ radiation due to flares. This emission can be confidently identified in the UVCS data because it produces both a simultaneous line brightening all along the UVCS slit and a characteristic O VI doublet intensity ratio $I(1032) / I(1037)=4: 1$. Raymond et al. (2007) conceived a technique that made use of the resonantly scattered coronal radiation observed during the impulsive phase to obtain estimates of the $\mathrm{O}$ VI and transition region luminosities. From the analysis of the differential Doppler dimming along the UVCS slit, this technique also allows an estimate to be made of the flow speed of the O VI emitting plasma during the impulsive phase of the flare. The work of Raymond et al. (2007), who analyzed five GOES X-class flares observed during solar maximum activity with UVCS at $1.63 R_{\odot}$, was later extended by Johnson et al. (2011) through the analysis of an additional 24 events (Bthrough X-class) occurring between 1998 and 2005. The events selected in the two previous works were found during a search of the online CDAW LASCO CME catalog ${ }^{1}$ for events where the UVCS slit was positioned roughly over the flare. Only those

\footnotetext{
1 http://cdaw.gsfc.nasa.gov/CME_list/
} 
flare events that were clearly associated with coronal mass ejections (CMEs) were included. When a CME reaches the position of the UVCS slit, it blows the pre-event corona away so that the intensity of the scattered O VI photons rapidly declines. Thus, in the presence of CMEs, this technique provides intensities only for 5 to $15 \mathrm{~min}$ in the impulsive phase of the flare. In this work, we were able to directly search in the UVCS database, thus including flares that have no apparent associated CME signature in white light, plus a few other events that were missed in the previous searches, bringing the total number of $\mathrm{O}$ VI flares to 58 .

The observations are presented in Sect. 2. We analyze the data and interpret the results in Sect. 3. A summary and conclusions are presented in Sect. 4.

\section{Observations}

\subsection{UV observations}

The Ultraviolet Coronagraph Spectrometer (UVCS) is a long-slit UV spectrograph consisting of two spectrometric channels (OVI and LYA) for the observation of spectral lines in the UV range with an instantaneous field of view of $40^{\prime}$ on a line parallel to a tangent to the solar limb with nominal spatial resolution of $7^{\prime \prime}$ per pixel. The UVCS telescope mirror and instrument rotation mechanisms can point the spectrograph slits to observe anywhere in the corona at heliocentric distances between about 1.4 and $10 R_{\odot}$. Intensities for the O VI 1032, $1037 \AA$ spectral lines were measured using the latest UVCS Data Display and Analysis Software, Version 5.1 (DAS51). This software removes image distortion, and calibrates the data in wavelength and intensity taking into account the time dependence of the instrument performance.

We analyzed a total of 58 events, 21 of which previously studied by Johnson et al. (2011). These events were reanalyzed for the purpose of consistency because the new analysis was performed with more accurate calibration techniques. We note that we discovered some severe inconsistencies while reanalyzing the data included in that work. In particular, we found that most of the values obtained for the soft X-ray emission measures in the events analyzed by Johnson et al. (2011) were overestimated by a factor of about five. As a consequence, the X-ray luminosities were overestimated by a similar factor. It has been suggested (anonymous referee) that the difference might be explained by the use of the earlier routine published by Thomas et al. (1985) for calculating the temperature and emission measure from the GOES 2-channel X-ray fluxes. The new routine published by White et al. (2005), now used in the IDL GOES Workbench, gives a smaller emission measure than the one given by the older routine - by a factor of about four - that is, however, still lower than the factor of five reported above. The reason for the discrepancies with the present work is unfortunately not known since a detailed reconstruction of the data reduction and analysis procedures used in the Johnson et al. (2011) paper is no longer available; therefore, we chose to reanalyze the raw data for all the events using the most recent calibration routines to date. We are confident that the present results are more reliable than those previously reported.

We started searching for all the O VI flare events in the online UVCS CME catalog (Giordano et al. 2013) ${ }^{2}$, which provides a preliminary categorization of the observed features related to the CMEs detected by UVCS. In particular, there are some tens of events clearly or possibly identified as $\mathrm{O}$ VI flares. Not all

\footnotetext{
2 Available at the URL http://solarweb.oato.inaf.it/UVCS_ CME
}

the observed O VI flares are related to an observed CME, and so we conducted a systematic search through the entire ten-year UVCS data set, spanning from 1996 to 2005. Based on the list of X-ray flares detected by GOES satellites that is available at the NGDC/NOAA events catalogs ${ }^{3}$, we selected the limb events located at a projected distance $\geq 0.9 R_{\odot}$ from the Sun center. For each given event, we searched for the UVCS data at the time of the X-ray flare peak in the polar angle region of $\pm 15^{\circ}$ above the flare position. We only selected UVCS observations with exposure times $\leq 300 \mathrm{~s}$, which allow the time profile of the O VI luminosity to be determined with enough temporal resolution. The observation parameters for each of the events analyzed in this paper are listed in Table A.1 where we give the GOES X-ray class, the central position angle (PA) and height of the UVCS slit, the exposure time, the slit width, and the estimated Doppler speed $\left(V_{\mathrm{dop}}\right)$ for each event. The central position angle is defined as the angular position of the center of the slit measured in the radial direction normal to the slit counterclockwise (CCW) from the heliographic north. Figure 1 shows an example UVCS observation for an M 1.5 class event, the X-ray flare of March 20, 2003. The middle panel shows the brightness of the O VI 1032 line as a function of spatial position along the slit (horizontal direction covering position angles between $221^{\circ}$ and $285^{\circ}$ ) and time (vertical). The pre-event background emission was subtracted. The O VI brightening, appearing at about 11:30 UT, also visible in the right hand panel of the same figure, results from $O$ VI photons scattered from $\mathrm{O}$ VI ions in the corona.

When the CME reaches the UVCS slit, it reduces the scattered O VI brightness because the Doppler dimming effect drastically reduces the scattering rate and because there is little $\mathrm{O}$ VI in the hot plasma. We went through the list of the events presented in Johnson et al. (2011) and calculated the times at which the CMEs were supposed to reach the slit by using the constant CME speed estimates obtained from the online CDAW LASCO $\mathrm{CME}$ catalog. These are clearly lower limits since the CMEs are often accelerating within the first few solar radii, so that the initial speed is probably lower. For 7 out of the 29 events analyzed by Johnson et al. (2011) we suspect that the associated CMEs might have reached the slit before or during the enhancement seen in the $\mathrm{O}$ VI spectral lines. In those cases, the measured lag time between the O VI and X-ray peaks must be considered as an upper limit and the transition region luminosities are to be considered as lower limits. Another event analyzed in that paper (on 2001 December 28) does not show a clear association with the soft-X ray flare. These eight events from Johnson et al. (2011) have thus been excluded in the following analysis.

\subsection{Soft-X-ray observations}

The soft X-ray observations used in this work were obtained with the GOES satellite that measures the soft X-ray flux in two broadband wavelength intervals (1-8 $\AA$ and $0.5-4 \AA$ ). GOES observations can be used to compute isothermal temperatures, $T_{\mathrm{X}}$, and emission measures, defined as $\mathrm{EM}_{\mathrm{X}} \equiv \int n_{E}^{2} \mathrm{~d} V$, as a function of time. The X-ray flux, $F_{\mathrm{X}}$, measured in either of the two GOES channels, is given by $F_{\mathrm{X}} \propto G\left(T, n_{\mathrm{e}}\right) \mathrm{EM}_{\mathrm{X}} \mathrm{W} / \mathrm{m}^{2}$. In the above relation, $G\left(T, n_{\mathrm{e}}\right)$ is the contribution function for an isothermal plasma at temperature $T_{\mathrm{X}}$ and electron density $n_{\mathrm{e}}$. Techniques have been developed to derive $T_{\mathrm{X}}$ and $\mathrm{EM}_{\mathrm{X}}$ from the ratio of the long and short channels (e.g., Thomas et al. 1985; Garcia 1994; White et al. 2005). These techniques rely on the fact that the ratio of the X-ray intensities seen through

\footnotetext{
ftp://ftp.ngdc.nasa.gov/STP/SOLAR_DATA
} 

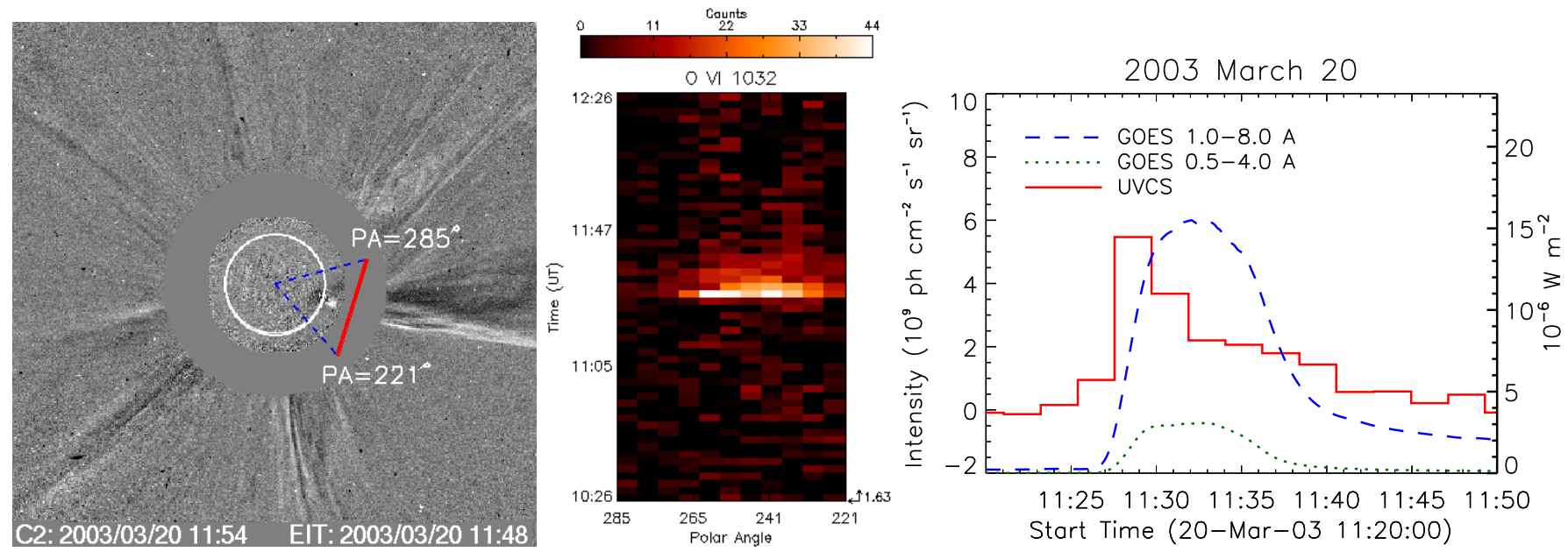

Fig. 1. Left: LASCO and EIT difference images at the time of the GOES M1.5 class flare of 2003 March 20 with the UVCS slit position superimposed. Middle: Brightness of the O VI 1032 line as a function of spatial position along the slit (horizontal direction) and time (vertical direction). The pre-event background was subtracted. Right: O VI 1032 A intensity after subtraction of the pre-event intensity (red histogram) plotted against time for the same event. The GOES soft X-ray flux is plotted as a blue dashed line. The GOES hard X-ray flux is shown with the green dotted line. The left-hand scale applies to O VI, and the right-hand scale to GOES.

two filters with different wavelength bandpasses is a function of the plasma temperature alone if one assumes emission from an isothermal plasma. X-ray temperatures $T_{\mathrm{X}}$ can be thus inferred from the observed intensity ratios by comparison with synthetic spectra, here determined from version 7 of the CHIANTI atomic database (e.g., Dere et al. 1997, 2009; Landi et al. 2012, 2013). Once the temperature of the plasma is estimated, its emission measure $\mathrm{EM}_{\mathrm{X}}$ can be determined from the intensity observed from the filter with the strongest signal. For each event, after subtracting the pre-flare fluxes from the observed light curves, we determined the time profiles of temperatures and emission measures of the soft X-ray flares corresponding to the O VI flares observed in Table A.1 using the IDL routine goes_chianti_tem distributed in the Solar Software Library (Freeland \& Handy 1998). Given the above parameters, the thermal luminosity of the X-ray emitting plasma was finally derived by applying the IDL routine calc_rad_loss using default coronal abundances.

\section{Analysis and results}

We derived the transition region luminosities during the impulsive phase of the flares listed in Table A.1 by following the steps outlined in the two previous works of Raymond et al. (2007) and Johnson et al. (2011). The O VI luminosity $L_{\mathrm{OVI}}$ for each exposure of the flare was inferred by using the estimate of the O VI column density obtained from pre-flare observations. Since $L_{\mathrm{OVI}} \propto \mathrm{Ab}(\mathrm{O}) \mathrm{EM}_{\mathrm{OVI}}$, the volume emission measure, defined as $\mathrm{EM}_{\mathrm{OVI}} \equiv \int n_{\mathrm{e}} n_{\mathrm{H}} \mathrm{d} V \mathrm{~cm}^{-3}$, was derived from the observed $\mathrm{O}$ VI luminosity given an estimate for the oxygen abundance $\mathrm{Ab}(\mathrm{O})$ relative to hydrogen. In this work, we use the solar photospheric abundances of Asplund et al. (2009) and typical transition region densities of $10^{11} \mathrm{~cm}^{-3}$. Recently, new calculations of the ionization and recombination rates have been published and are summarized in the CHIANTI database (Landi et al. 2013). This new computation results in significant differences with respect to the previous calculations by Mazzotta et al. (1998). In general, the $L_{\mathrm{TR}}$ values estimated in this work are higher by a factor of 1.46 than the ones reported in Johnson et al. (2011) for the same events. This is expected, since the Asplund et al. (2009) set of abundances have less oxygen $(\mathrm{Ab}(\mathrm{O})=8.69$ instead of $\mathrm{Ab}(\mathrm{O})=8.83$ given by Grevesse et al. 1998) and thus require larger emission measures. The temperature of maximum ionization for $\mathrm{O}$ VI is $\log T_{\max }(\mathrm{K})=5.47$. From $\mathrm{EM}_{\mathrm{OVI}}$, the luminosity $L_{\mathrm{TR}}$ of the transition region (here defined as the region with temperatures $5.0 \leq \log T(\mathrm{~K}) \leq 6.0)$ was finally estimated as in Raymond et al. (2007) by assuming that $\operatorname{EM}(\log T)$ scales with $T$ as appropriate for a thermal conduction boundary layer in equilibrium or an evaporative flow (Raymond \& Doyle 1981) and then multiplying by the cooling rates.

\subsection{Soft X-ray vs. UV relationships during flares}

Chromospheric evaporation, first proposed by Neupert (1968), is the process by which solar flares produce their high-temperature, high-density plasma. In the framework of the standard flare model (CSHKP; Carmichael 1964; Sturrock 1966; Hirayama 1974; Kopp \& Pneuman 1976), which involves the formation of a vertical flare current sheet behind an ejected flux rope/filament, magnetic reconnection in the current sheet is expected to initiate or allow mechanisms that can lead to electron acceleration. The accelerated electrons travel downward along the field lines to the chromosphere. If the atmosphere is unable to radiate away the energy deposited, Coulomb collisions with ambient electrons result in an overall heating and consequent expansion of the chromospheric material. In turn, the chromospheric evaporation fills the flaring loops with hot plasma up to a temperature of $\gtrsim 10^{7} \mathrm{~K}$, giving rise to soft X-ray emission.

During the impulsive phase of solar flares, the O VI emission is expected to arise from the rapidly heated plasma. This emission could be excited by the evaporating plasma that is heated at the temperatures of the post-flare loops and that expands upwards. Alternatively, it could be produced by chromospheric plasma heated by energetic particles or shocks in the footpoints. This is plasma that is heated but either remains stationary or moves because of the pressure of the gas above it. Redshifts and blueshifts are commonly observed in transition region lines during the impulsive phases of flares. Finally, this emission could be produced by the outward moving plasma in the ejected prominence. All three of these likely sources may contribute in different events or even in a single event, and the relative timing of UV and X-ray brightness peaks, the flow speeds, and the total 




Fig. 2. GOES peak flux plotted against the time lag $\Delta t \equiv t_{F_{\mathrm{X}} \text {,peak }}-$ $t_{F_{\mathrm{OVI}} \text {,peak }}$ between the peak of the $\mathrm{O}$ VI luminosity and the peak of the $\mathrm{X}$-ray flux. Empty circles represent the events for which CMEs were detected. The 22 events for which no associated CMEs were observed are indicated by filled circles.

O VI luminosity favor each source in one or more events. The correlations between X-ray and UV intensities expected in these three cases can be considered and thus compared to the observations. In the evaporation scenario, it is expected that the thermal $\mathrm{X}$-ray emission peaks after the emission from the evaporation flow as the loops fill with hot plasma, that is, when the chromospheric evaporation is nearly complete. The peak of the observed transition region emission should thus precede the peak observed in the soft X-rays for the same events. In Fig. 2, we show a plot of the GOES peak flux against the time lag $\Delta t \equiv t_{F_{\mathrm{X}} \text {,peak }}-t_{F_{\text {OVI }} \text {,eak }}$ from the O VI peak to the GOES peak flux for the 58 events analyzed in this work. Within the quoted uncertainties, the soft $\mathrm{X}$-ray flux peaks in a range between -3 and 15 min with respect to the peak of the $\mathrm{O}$ VI intensity, with a mean of $3.2 \pm 0.1 \mathrm{~min}$. Johnson et al. (2011) also found a loose correlation: larger flares usually have the GOES peak later than the O VI peak, while in smaller flares it can either lag or be simultaneous. This would be consistent with the idea that flares develop over time, with larger flares taking longer to energize the large number of the magnetic loops that become part of the flare. Our analysis, however, does not show a clear correlation $(r=0.24)$ and the scatter is substantial. From the same plot, we infer that most of the 22 events with no associated CME show $\Delta t>0$, within the quoted uncertainties, and that they can be thus interpreted in the frame of the evaporation or footpoint scenarios. For the ten events in which an associated CME was detected and for which $\Delta t \lesssim 0$, the presence of $\mathrm{O}$ VI emission from the erupting prominence is a more likely explanation. However, for the remaining events with associated CME and positive time lags, either interpretation is possible.

To test for a correlation between soft X-ray emission and the amount of evaporated material expected in the evaporation scenario, we compared the total transition region emission $E_{\mathrm{TR}}$ observed during the UVCS exposures corresponding to the peak $\mathrm{O}$ VI emission (i.e., $L_{\mathrm{TR}}$ times the exposure time) to the change in GOES X-ray luminosity $\Delta L_{\mathrm{X}}$ between the UVCS exposures corresponding to the peak $\mathrm{O}$ VI emission and the following UVCS exposure. The UVCS exposure time for each event, ranging from 75 to $300 \mathrm{~s}$ with a time between exposures of about $4 \mathrm{~s}$, are detailed in Table A.1. Only the cases for which $\Delta L_{X}>0$ are shown in the left panel of Fig. 3 (38 out of 58, or about two-thirds of the selected events). The solid line is a least-square linear fit to the log-log data given by $\log _{10}\left(\Delta L_{\mathrm{X}}\right)=a+b \cdot \log _{10}\left(E_{\mathrm{TR}}\right)$, with $a=5.4 \pm 9.4$ and $b=0.71 \pm 0.33$. We used these estimates, together with percentile points from the Student $t$ distribution with the same degrees of freedom as the regression to form a $95 \%$ confidence interval for the estimate of the slope (dotted lines in Fig. 3). According to the F statistic, there is significant evidence of a log-log relationship (the correlation coefficient is $r=0.59$ ).

There is empirical evidence that for many flares the soft $\mathrm{X}$-ray time derivative closely matches the hard X-ray time profile. This result, called the Neupert effect (Neupert 1968), suggests a causal relationship between nonthermal (hard X-ray or microwave) and thermal (soft X-ray) flare emissions. It follows that for the events in which the Neupert effect is observed there is evidence of chromospheric evaporation driven by accelerated electrons. The correlation between the X-ray luminosity and the UV energy is in agreement with the Neupert effect relationship. The observed scatter, however, hints to the fact that in the simple picture where the transition region emission originates from plasma evaporating to fill the X-ray loops, different factors such as the evaporation speed or the pressure may vary significantly from event to event. The 20 events showing a drop in $L_{\mathrm{X}}$ while $L_{\mathrm{TR}}$ is still high are unlikely in the evaporation scenario and might be interpreted in the light of the CME picture. The right panel of Fig. 3 shows the transition region luminosity $L_{\mathrm{TR}}$ during the peak UVCS exposure plotted against the GOES X-ray luminosity $L_{\mathrm{X}}$ at the peak of the $L_{\mathrm{TR}}$. The solid line is a least-square linear fit to the $\log$-log data given by $\log _{10}\left(L_{\mathrm{X}}\right)=$ $a+b \cdot \log _{10}\left(L_{\mathrm{TR}}\right)$, with $a=6.2 \pm 7.2$ and $b=0.75 \pm 0.27$. This plot also shows a correlation $(r=0.59)$ compatible with evaporation or footpoint origins. For CME emission, there are few systematic studies of transition region emission from erupting prominences during the early phases. However, since the amount of prominence material varies considerably among different events, the correlation between $L_{\mathrm{TR}}$ and $L_{\mathrm{X}}$ is likely to be loose. Moreover, most energetic X-ray events analyzed by UVCS show little or no cool gas seen in CMEs (e.g., Raymond et al. 2003, 2007), so the above correlations tend to disfavor an erupting prominence origin for the $\mathrm{O}$ VI emission. Overall, the results are thus consistent with transition region emission originating from chromospheric evaporation, where the thermal X-ray emission peaks after the emission from the evaporation flow as the loops fill with hot plasma.

\subsection{Flow speed estimates}

The evaporation velocity has traditionally been detected through Doppler measurements of EUV and soft X-ray emission lines. Several spectroscopic observations have disclosed the plasma dynamics in the early phase of the flare, showing upflows of up to a few hundred $\mathrm{km} \mathrm{s}^{-1}$ in hot lines formed at a few million degrees (e.g., Doschek et al. 1980, 1992; Canfield et al. 1987). On the other hand, in chromospheric and transition region lines, redshifts of around tens of $\mathrm{km} \mathrm{s}^{-1}$ were detected, implying downflows (Wuelser et al. 1994; Czaykowska et al. 1999; Teriaca et al. 2003, 2006; Brosius 2003; Del Zanna et al. 2006). The evaporation is said to be gentle when the emission lines formed in the upper chromosphere, transition region and hot corona appear blueshifted by several tens of $\mathrm{km} \mathrm{s}^{-1}$, with no associated downflows. When the lines formed in the upper chromosphere and transition region appear redshifted by several tens of $\mathrm{km} \mathrm{s}^{-1}$, while lines with higher formation temperatures appear blueshifted by hundreds of $\mathrm{km} \mathrm{s}^{-1}$, the evaporation is said to be explosive (Fisher et al. 1985). The redshifts are supposedly due to chromospheric condensation caused by the pressure gradient induced by the violent heating of the 

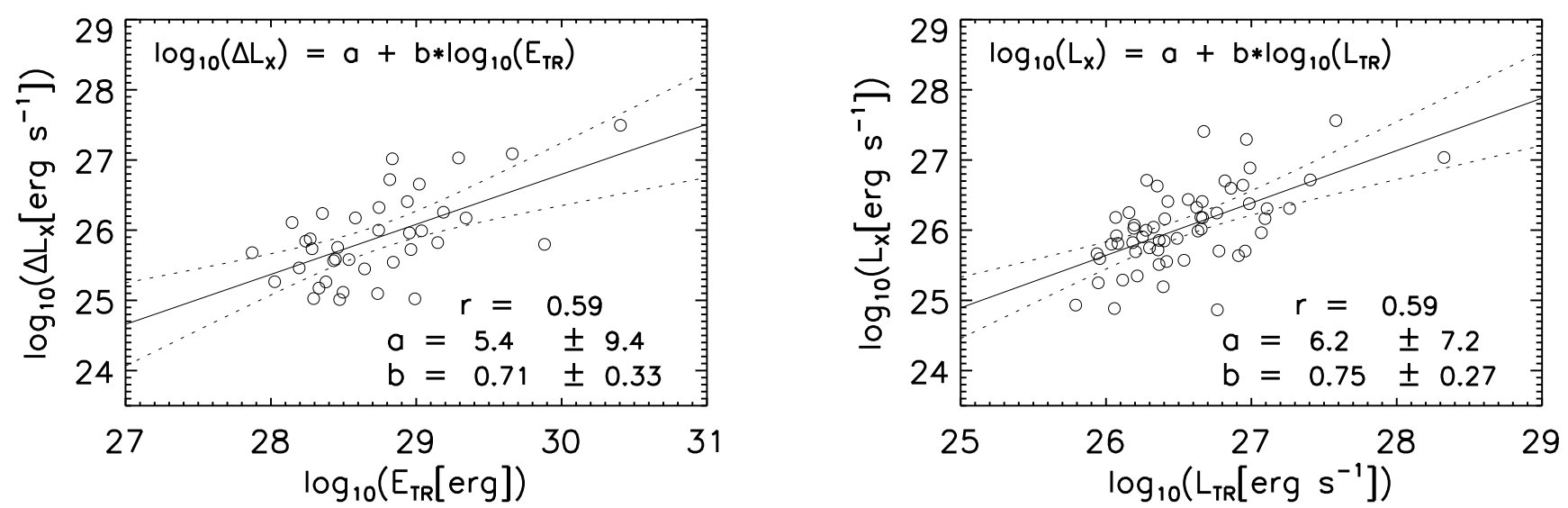

Fig. 3. Soft X-ray vs. UV flare relationships. Left: total transition region emission $E_{\mathrm{TR}}$ observed during the UVCS exposures corresponding to the peak O VI emission (i.e., $L_{\mathrm{TR}}$ times the exposure time), plotted against the variation in GOES X-ray luminosity $\Delta L_{\mathrm{X}}$ between the UVCS exposures corresponding to the peak O VI emission and the following exposure. Right: transition region luminosity $L_{\mathrm{TR}}$ during the peak UVCS exposure plotted against the GOES X-ray luminosity $L_{\mathrm{X}}$ at the peak of the $L_{\mathrm{TR}}$. Also shown are the least-square linear fits to the log-log data (solid line), i.e., the best fit power-law regressions, together with the respective $95 \%$ confidence intervals (dotted lines).

chromosphere or the transition region produced by the energetic electrons. At transition region temperatures around $10^{5} \mathrm{~K}$, both upflows and downflows have been reported in previous studies. Spectroscopic observations by missions such as the Coronal Diagnostic Spectrometer (CDS; Harrison et al. 1995) and the EUV Imaging Spectrometer (EIS; Culhane et al. 2007) have also identified these dynamical phenomena in UV and EUV lines at the footpoints of flare loops (e.g., Milligan et al. 2006; Milligan \& Dennis 2009). EIS was used to measure Doppler shifts in 15 emission lines covering the temperature range $T=0.05-16 \mathrm{MK}$ during the impulsive phase of a C-class flare. Blueshifts, indicative of the evaporated material, were observed in Fe XIV-Fe XXIV emission lines (2-16 MK) while emission from $\mathrm{O}$ VI-Fe XIII lines $(0.5-1.5 \mathrm{MK})$ was found to be redshifted by $v \approx 60-17 \cdot T \mathrm{~km} \mathrm{~s}^{-1}$, with $T$ in MK, and was interpreted as the downward-moving "plug characteristic" of explosive evaporation (Milligan \& Dennis 2009). The dependence of velocity on temperature of evaporating material has been predicted by Fisher et al. (1985): chromospheric material heated to different temperatures is subject to different pressure gradients relative to the overlying corona and therefore it rises at different rates.

Doppler dimming can cause the derived illuminating flux to vary along the slit. The Doppler dimming effect produced by the chromospheric evaporation can be constrained because the flow should be directed toward the portion of the UVCS slit directly above the flare, while the Doppler shift affecting the different points along the slit must be reduced by a factor corresponding to the cosine of the angle from the vertical. In order to estimate the flow speed $V_{\text {dop }}$, within a range of about $20 \mathrm{~km} \mathrm{~s}^{-1}$ (Johnson et al. 2011), we assumed that the value of the derived luminosity $L_{\text {flare }}(1032)$ should be as uniform as possible for the portions of the UVCS slit where the signal-to-noise ratio was significant. For all events, we used eight segments, a number of slit portions large enough to provide a signal-to-noise ratio of at least three to one. Flow speeds in the $20-100 \mathrm{~km} \mathrm{~s}^{-1}$ range were observed for 19 out of 58 events, with 14 of them showing speeds between 60 and $80 \mathrm{~km} \mathrm{~s}^{-1}$. These values are compatible with the ones found with direct spectroscopic Hinode EIS observations at transition region temperatures for the redshifted O VI emission from the flare footpoints by Milligan \& Dennis (2009). Unfortunately, the Doppler dimming technique used in this work is only able to yield the magnitude of the observed flow speed and does not allow to distinguish between up- and downflows. We also remark that possible flow speeds $>100 \mathrm{~km} \mathrm{~s}^{-1}$ may remain undetected since the O VI emission would be severely Doppler dimmed for high speed plasma and that the Doppler dimming technique is not sensitive to flow speeds $<20 \mathrm{~km} \mathrm{~s}^{-1}$. (e.g., Kohl et al. 1995).

\section{Summary and conclusions}

The nature of the emission from UV spectral lines generated from flares in the transition region is poorly known as yet owing to the limited field of view of the spectrographs and because the impulsive phase usually occurs in a small region and only lasts a short time. In some favorable cases, however, such as near-limb events, the flare's signature can be detected indirectly in observations of resonantly scattered radiation from $\mathrm{O}$ VI ions higher up in the corona. In this work, we derived the O VI $1032 \AA$ luminosity profiles of 58 flares during their impulsive phase and observed off-limb by the UVCS/SOHO spectrograph from 1997 to 2005. The UV luminosities from the transition region plasma were inferred from the analysis of the resonantly scattered radiation of the $\mathrm{O}$ VI coronal ions. Comparison of spectroscopic UV observations with simultaneous soft X-ray measurements has been used to investigate the temporal relationship between flare mechanisms that produce UV and X-ray emission. Specifically, this analysis provides a means to pinpoint the likely source (chromospheric evaporation, footpoint emission, or heated prominence ejecta) for the transition region emission observed during the impulsive phase of the flares.

The impulsive-phase UV emission, most likely due to accelerated electrons impacting the chromosphere, should precede the more slowly evolving soft X-ray emission produced by the heated plasma. In agreement with the Neupert effect relationship, if we adopt the evaporation picture, we expect a correlation between the X-ray luminosity and the UV energy, suggesting that the plasma is heated from the footpoints to the loop top, first in the chromosphere and only a few minutes later in the corona. In our study, we find evidence of both features predicted by the evaporation scenario. First, most O VI flares precede the X-ray peaks, typically by several minutes with a mean of $3.2 \pm 0.1 \mathrm{~min}$. Second, we find correlations between the soft 
$\mathrm{X}$-ray and transition region luminosities following power laws with indices $\sim 0.7 \pm 0.3$ (Fig. 3). The results are thus consistent with transition region emission originating from chromospheric evaporation: the thermal X-ray emission peaks after the emission from the evaporation flow as the loops fill with hot plasma. Although O VI emission from erupting prominences cannot be excluded in the cases where a CME eruption was observed in white light, it is more likely for the ten events in which an associated CME was detected and the GOES X-ray peak preceded the O VI flare peak.

Finally, for 19 events (see Table A.1), we were able to infer flow speeds in the range $20-100 \mathrm{~km} \mathrm{~s}^{-1}$, of which 14 showed speeds between 60 and $80 \mathrm{~km} \mathrm{~s}^{-1}$. These values are compatible in magnitude with those found through direct spectroscopic Hinode EIS observations at transition region temperatures by Milligan \& Dennis (2009).

Acknowledgements. We thank the referee for providing constructive comment and help in improving the contents of this paper. This work was made possible by the UVCS/SOHO instrument and Flight Operations team. UVCS is a joint project of the National Aeronautics and Space Administration (NASA), the Agenzia Spaziale Italiana (ASI) and Swiss founding agencies.

\section{References}

Asplund, M., Grevesse, N., Sauval, A. J., \& Scott, P. 2009, ARA\&A, 47, 481 Brosius, J. W. 2003, ApJ, 586, 1417

Canfield, R. C., Metcalf, T. R., Strong, K. T., \& Zarro, D. M. 1987, Nature, 326, 165

Canfield, R. C., Metcalf, T. R., Zarro, D. M., \& Lemen, J. R. 1990, ApJ, 348, 333

Carmichael, H. 1964, NASA SP, 50, 451

Culhane, J. L., Harra, L. K., James, A. M., et al. 2007, Sol. Phys., 243, 19

Czaykowska, A., de Pontieu, B., Alexander, D., \& Rank, G. 1999, ApJ, 521, L75

Del Zanna, G., Berlicki, A., Schmieder, B., \& Mason, H. E. 2006, Sol. Phys., 234,95
Dere, K. P., Landi, E., Mason, H. E., Monsignori Fossi, B. C., \& Young, P. R. 1997, A\&AS, 125, 149

Dere, K. P., Landi, E., Young, P. R., et al. 2009, A\&A, 498, 915

Doschek, G. A., Feldman, U., Kreplin, R. W., \& Cohen, L. 1980, ApJ, 239, 725

Doschek, G. A., Mariska, J. T., Watanabe, T., et al. 1992, PASJ, 44, L95

Fisher, G. H., Canfield, R. C., \& McClymont, A. N. 1985, ApJ, 289, 414

Freeland, S. L., \& Handy, B. N. 1998, Sol. Phys., 182, 497

Garcia, H. A. 1994, Sol. Phys., 154, 275

Giordano, S., Ciaravella, A., Raymond, J. C., et al. 2013, J. Geophys. Res., 118, 967

Grevesse, N., \& Sauval, A. J. 1998, in Solar Composition from Core to Corona, eds. C. Frohlich, et al. (Dordrecht: Kluwer), 161

Grevesse, N., Asplund, M., Sauval, A. J., Scott, P. 2010, Ap\&SS, 328, 179

Harrison, R. A., Sawyer, E. C., Carter, M. K., et al. 1995, Sol. Phys., 162, 233 Hirayama, T. 1974, Sol. Phys., 34, 323

Kohl, J. L., Esser, R., Gardner, L. D., et al. 1995, Sol. Phys., 162, 313

Kohl, J. L., Noci, G., Antonucci, E., et al. 1997, Sol. Phys., 175, 613

Kopp, R. A., \& Pneuman, G. W. 1976, Sol. Phys., 50, 85

Johnson, H., Raymond, J. C., Murphy, N. A., et al. 2011, ApJ, 735, 70

Landi, E., Del Zanna, G., Young, P. R., Dere, K. P., \& Mason, H. E. 2012, ApJ, 744, 99

Landi, E., Young, P. R., Dere, K. P., Del Zanna, G., \& Mason, H. E. 2013, ApJ, 763, 86

Mazzotta, P., Mazzitelli, G., Colafrancesco, S., \& Vittorio, N. 1998, A\&AS, 133, 403

Milligan, R. O., \& Dennis, B. R. 2009, ApJ, 699, 968

Milligan, R. O., Gallagher, P. T., Mathoudiakis, M., et al. 2006, ApJ, 638, L117

Neupert, W. M. 1968, ApJ, 153, L59

Noci, G., Kohl, J. L., \& Withbroe, G. W. 1987, ApJ, 315, 706

Raymond, J. C., \& Doyle, J. G. 1981, ApJ, 247, 686

Raymond, J. C., Kohl, J. L., Noci, G., et al. 1997, Sol. Phys., 175, 645

Raymond, J. C., Ciaravella, A., Dobrzycka, D., et al. 2003, ApJ, 597, 1106

Raymond, J. C., Holman, G., Ciaravella, A., et al. 2007, ApJ, 659, 750

Sturrock, P. A. 1966, Nature, 211, 695

Teriaca, L., Falchi, A., Cauzzi, G., et al. 2003, ApJ, 588, 596

Teriaca, L., Falchi, A., Falciani, R., Cauzzi, G., \& Maltagliati, L. 2006, A\&A, 455,1123

Thomas, R. J., Crannell, C. J., \& Starr, R. 1985, Sol. Phys., 95, 323

White, S. M., Thomas, R. J., \& Schwartz, R. A. 2005, Sol. Phys., 227, 231

Woods, T. N., Tobiska, W. K., Rottman, G. J., \& Worden, J. R. 2000, J. Geophys. Res., 105, 12

Wuelser, J.-P., Canfield, R. C., Acton, L. W., et al. 1994, ApJ, 424, 459 
S. Mancuso et al.: Coronal O VI emission observed with UVCS/SOHO during solar flares

\section{Appendix A}

Table A.1. UVCS solar flare observations.

\begin{tabular}{|c|c|c|c|c|c|c|c|c|}
\hline $\begin{array}{c}\text { Date } \\
\text { (yyyy/mm/dd) }\end{array}$ & $\mathrm{CME}^{a}$ & $\begin{array}{c}\text { Flare peak } \\
(\text { UT) }\end{array}$ & X-ray class & $\begin{array}{c}\mathrm{PA} \\
\left({ }^{\circ} \mathrm{CCW}\right) \\
\end{array}$ & $\begin{array}{c}\text { Slit height } \\
\left(R_{\odot}\right)\end{array}$ & $\begin{array}{c}\text { Exposure } \\
(\mathrm{s})\end{array}$ & $\begin{array}{c}\text { Slit } \\
(\mu \mathrm{m})\end{array}$ & $\begin{array}{c}V_{\mathrm{dop}} \\
\left(\mathrm{km} \mathrm{s}^{-1}\right) \\
\end{array}$ \\
\hline $1997 / 03 / 06$ & $\mathrm{y}$ & $00: 49$ & В 8.9 & 90 & 1.55 & 200 & 49 & \\
\hline $1998 / 01 / 20^{b}$ & $\mathrm{y}$ & $19: 40$ & C 1.5 & 60 & 1.67 & 200 & 50 & \\
\hline $1998 / 04 / 06$ & $\mathrm{y}$ & $16: 48$ & M 1.1 & 120 & 1.56 & 200 & 49 & 20 \\
\hline $1998 / 12 / 13$ & $\mathrm{n}$ & $07: 12$ & C 5.5 & 270 & 1.69 & 100 & 101 & \\
\hline $1999 / 05 / 17$ & $\mathrm{y}$ & 18:03 & C 5.6 & 250 & 1.43 & 200 & 77 & \\
\hline $1999 / 08 / 02$ & $\mathrm{y}$ & $21: 25$ & X 1.4 & 225 & 1.55 & 75 & 49 & 30 \\
\hline $1999 / 12 / 26^{b}$ & $\mathrm{n}$ & $19: 24$ & C 2.7 & 249 & 1.49 & 100 & 150 & 70 \\
\hline $1999 / 12 / 28^{b}$ & $\mathrm{y}$ & $00: 48$ & M 4.5 & 249 & 2.58 & 100 & 98 & 60 \\
\hline $2000 / 02 / 05^{b}$ & $\mathrm{y}$ & $19: 28$ & X 1.2 & 102 & 1.67 & 120 & 49 & \\
\hline $2000 / 02 / 06^{b}$ & $\mathrm{n}$ & $16: 48$ & C 2.4 & 102 & 1.68 & 120 & 49 & 70 \\
\hline $2000 / 05 / 02^{b}$ & $\mathrm{n}$ & $01: 13$ & C 4.1 & 255 & 2.34 & 120 & 50 & \\
\hline $2000 / 05 / 23$ & $\mathrm{y}$ & $17: 54$ & C 4.3 & 245 & 2.34 & 120 & 48 & \\
\hline $2000 / 06 / 23$ & $\mathrm{y}$ & $14: 31$ & M 3.0 & 290 & 2.32 & 120 & 48 & \\
\hline $2000 / 09 / 24^{b}$ & $\mathrm{y}$ & $21: 54$ & C 5.0 & 110 & 1.64 & 120 & 97 & \\
\hline $2000 / 09 / 30^{b}$ & $\mathrm{y}$ & $17: 29$ & C 2.7 & 100 & 1.63 & 120 & 97 & \\
\hline $2000 / 10 / 25$ & $\mathrm{y}$ & 23:01 & C 1.8 & 100 & 1.63 & 120 & 98 & \\
\hline $2000 / 10 / 26$ & $\mathrm{y}$ & $16: 11$ & C 8.5 & 100 & 1.63 & 120 & 98 & \\
\hline $2001 / 01 / 05$ & $\mathrm{n}$ & $18: 34$ & C 6.8 & 90 & 2.34 & 75 & 48 & 70 \\
\hline $2001 / 03 / 24^{b}$ & $\mathrm{y}$ & $19: 55$ & M 1.7 & 16 & 2.12 & 300 & 77 & 90 \\
\hline $2001 / 06 / 13^{b}$ & $\mathrm{y}$ & $16: 28$ & C 9.1 & 263 & 1.62 & 120 & 100 & 60 \\
\hline 2001/08/07 & $\mathrm{n}$ & $00: 13$ & C 3.1 & 245 & 1.63 & 120 & 97 & \\
\hline 2001/08/07 & $\mathrm{y}$ & $16: 28$ & C 3.6 & 245 & 1.63 & 120 & 96 & \\
\hline $2001 / 08 / 07^{b}$ & $\mathrm{y}$ & 20:00 & C 1.9 & 245 & 1.63 & 120 & 96 & \\
\hline $2001 / 08 / 07^{b}$ & $\mathrm{n}$ & $23: 55$ & C 1.8 & 245 & 1.63 & 120 & 96 & \\
\hline $2001 / 08 / 11^{b}$ & $\mathrm{y}$ & $01: 21$ & C 5.2 & 290 & 1.61 & 120 & 97 & \\
\hline 2001/08/11 & $\mathrm{y}$ & 01:38 & С 2.9 & 290 & 1.61 & 120 & 97 & \\
\hline $2001 / 08 / 11$ & $\mathrm{n}$ & $11: 49$ & C 1.2 & 290 & 1.61 & 120 & 97 & \\
\hline $2001 / 08 / 11$ & $\mathrm{n}$ & $12: 27$ & C 1.3 & 290 & 1.61 & 120 & 97 & \\
\hline $2001 / 08 / 11^{b}$ & $\mathrm{y}$ & $17: 26$ & В 7.7 & 290 & 1.61 & 120 & 97 & \\
\hline $2001 / 08 / 11^{b}$ & $\mathrm{n}$ & $23: 15$ & В 8.6 & 290 & 1.61 & 120 & 97 & 70 \\
\hline $2001 / 08 / 12^{b}$ & $\mathrm{y}$ & $17: 14$ & C 1.6 & 290 & 1.61 & 120 & 97 & 60 \\
\hline $2001 / 09 / 15$ & $\mathrm{y}$ & $11: 28$ & M 1.5 & 225 & 1.73 & 120 & 96 & \\
\hline $2001 / 12 / 10^{b}$ & $\mathrm{n}$ & $23: 48$ & C 7.0 & 263 & 1.57 & 200 & 96 & \\
\hline $2001 / 12 / 11^{b}$ & $\mathrm{y}$ & $14: 51$ & M 1.3 & 263 & 1.92 & 200 & 96 & \\
\hline $2001 / 12 / 29$ & $\mathrm{y}$ & $09: 45$ & M 9.3 & 282 & 1.90 & 200 & 98 & 20 \\
\hline $2002 / 01 / 06$ & $\mathrm{n}$ & $06: 52$ & C 5.3 & 270 & 1.57 & 200 & 98 & \\
\hline $2002 / 04 / 16$ & $\mathrm{y}$ & $10: 44$ & C 9.3 & 290 & 1.61 & 120 & 96 & \\
\hline $2002 / 04 / 17$ & n & $00: 40$ & С 9.9 & 290 & 1.61 & 120 & 96 & \\
\hline $2002 / 07 / 18$ & $\mathrm{y}$ & $07: 44$ & X 1.8 & 360 & 2.07 & 120 & 150 & 100 \\
\hline $2002 / 08 / 11$ & $\mathrm{y}$ & $14: 13$ & C 2.3 & 70 & 1.63 & 120 & 97 & 80 \\
\hline $2002 / 08 / 23$ & $\mathrm{n}$ & $04: 20$ & C 9.4 & 260 & 1.63 & 120 & 96 & 80 \\
\hline $2002 / 11 / 18$ & $\mathrm{n}$ & $07: 11$ & C 2.2 & 288 & 1.50 & 200 & 149 & \\
\hline $2003 / 03 / 20$ & $\mathrm{n}$ & $11: 31$ & M 1.5 & 253 & 1.63 & 120 & 102 & 60 \\
\hline $2003 / 06 / 01$ & $\mathrm{n}$ & $16: 52$ & M 1.4 & 263 & 1.68 & 120 & 98 & \\
\hline $2003 / 06 / 02$ & $\mathrm{y}$ & $15: 44$ & C 9.0 & 263 & 1.68 & 120 & 98 & 70 \\
\hline $2003 / 06 / 17$ & $\mathrm{n}$ & 01:02 & C 1.5 & 100 & 1.69 & 120 & 98 & \\
\hline $2003 / 11 / 05$ & $\mathrm{n}$ & $02: 41$ & M 1.6 & 262 & 1.67 & 120 & 96 & 70 \\
\hline $2003 / 11 / 05$ & $\mathrm{n}$ & $10: 52$ & M 5.3 & 262 & 1.67 & 120 & 96 & \\
\hline $2004 / 07 / 14$ & $\mathrm{n}$ & $20: 20$ & C 3.6 & 283 & 1.77 & 120 & 98 & \\
\hline $2004 / 07 / 14$ & $\mathrm{n}$ & $20: 54$ & C 6.6 & 283 & 1.77 & 120 & 98 & \\
\hline $2004 / 07 / 16$ & $\mathrm{y}$ & $13: 55$ & X 3.6 & 90 & 3.00 & 120 & 200 & \\
\hline $2004 / 07 / 27$ & $\mathrm{y}$ & $05: 45$ & M 1.1 & 270 & 1.77 & 120 & 99 & \\
\hline $2004 / 07 / 27^{b}$ & $\mathrm{y}$ & $15: 07$ & C 3.0 & 270 & 1.77 & 120 & 99 & \\
\hline $2004 / 07 / 27$ & $\mathrm{y}$ & $15: 25$ & C 2.8 & 270 & 1.77 & 120 & 99 & \\
\hline $2004 / 07 / 28^{b}$ & $\mathrm{y}$ & $21: 33$ & C 1.6 & 270 & 1.77 & 120 & 99 & \\
\hline $2004 / 10 / 05^{b}$ & $\mathrm{y}$ & $23: 17$ & B 9.0 & 75 & 1.72 & 120 & 98 & 60 \\
\hline $2004 / 11 / 03$ & $\mathrm{y}$ & $01: 33$ & M 2.8 & 310 & 1.71 & 120 & 98 & 60 \\
\hline $2005 / 07 / 10$ & $\mathrm{y}$ & $15: 16$ & C 9.9 & 284 & 2.42 & 120 & 76 & \\
\hline
\end{tabular}

Notes. ${ }^{(a)}$ Information on the presence ("y") or absence ("n") of an associated white light CME. (b) Events that have been already analyzed by Johnson et al. (2011). 